\title{
EDUCACIÓN PARA LA IGUALDAD EN TIEMPOS DE CRISIS
}

\author{
Iratxe Suberviola Ovejas \\ Universidad de La Rioja
}

RESUMEN: En este artículo se realiza una revisión desde dos vertientes: Por un lado, sobre la realidad desigual de las mujeres en el ámbito sociolaboral y, por otro, sobre las modificaciones que el mercado del trabajo está experimentando en estos momentos de crisis. Me apoyo en ambas directrices como fundamentación para realizar una revisión de los tradicionales roles de hombres y mujeres y proponer la modificación de los mismos a través de una educación igualitaria. Con este objeto se realiza un análisis de la situación actual de la mujer y de las prácticas coeducativas existentes, planteando cambios al respecto.

Palabras clave: Coeducación, mujer, educación de la mujer, roles sociales.

\section{EDUCATION FOR THE EQUALITY IN TIMES OF CRISIS}

ABSTRACT: This article realizes a review from two slopes: On the one hand, on the inequality of the women in the socio-labor area and, on the other hand, on the modifications that the labor market is experimenting on these moments. From these analyses there is based the review of the roles of men and women and one proposes the modification of these roles across the education for the equality. With this object there is realized an analysis of the current situation of the woman and of the coeducational existing practices, raising changes in the matter.

Keywords: Coeducation, woman, women's education, social roles.

Toda crisis supone una inestabilidad en diversos ámbitos que incide de manera desigual en las personas dependiendo de su nivel socio-económico, 
educativo, su época vital y también su sexo. Muchos de los cambios sociales surgen de momentos de no-acomodación como el actual. Desde la perspectiva de género y debido a los cambios producidos en el mercado laboral que incide directamente en los roles tradicionales, el ámbito educativo debe rentabilizar esta situación instrumentalizando la cambiante realidad.

La actual crisis que estamos padeciendo en España, surgió en el 2007 en EEUU. En nuestro país la economía se ha basado en los últimos años en la construcción, que ha permitido poner en circulación una importante suma de dinero especulativo a través del desenfreno de la misma. Partiendo de la necesidad básica de accesibilidad a la vivienda de los ciudadanos que obliga a cada individuo u familia a su adquisición, las trabajadoras y trabajadores salen perjudicados. Bajo el supuesto incremento indefinido de la vivienda, se concedieron hipotecas elevadas sin demasiados problemas o garantías de recuperación y préstamos a tipos muy bajos. Cuando el precio de la vivienda empezó a caer se produjo una alta preocupación entre las entidades financieras que vislumbraban la problemática que se avecinaba. Esto Ilevo a crear una desconfianza entre ellas, abortando los préstamos entre sí. De este modo, la crisis se fue convirtiendo en una crisis financiera internacional. Al dejar de fluir los créditos se cerro de golpe la época del dinero fácil y tanto familias como empresas empezaron a tener problemas para conseguir financiación 1 .

Desde una perspectiva optimista se debe destacar que toda crisis representa una oportunidad de cambios para innovar y crecer; de hecho, nos encontramos en un momento idóneo para realizar replanteamientos generales. Se aprecia un consenso en el análisis de que el modelo de desarrollo basado de forma exclusiva en el crecimiento económico no garantiza, ni la cohesión social, ni el desarrollo social de bienestar, ni el avance de una sociedad igualitaria ${ }^{2}$.

Desde la perspectiva de género, es necesario pararse a examinar algunos aspectos de la crisis que resultan claves como es el impacto en el empleo de ambos sexos, en la pobreza y en el trabajo doméstico y de cuidados no remunerados. En definitiva, conviene realizar un análisis sobre el mercado laboral de la mujer y los antecedentes que lo han producido.

Si se realiza un viaje por la evolución de la incorporación de la mujer en el mercado laboral se puede postular que la situación en el trabajo de éstas, hoy es el resultado de una incorporación desigual desde sus inicios. Parte de la responsabilidad de la discriminación laboral que hoy sufrimos las mujeres tienen su base en el ideal del "salario familiar" que implica que el sueldo del hombre debe ser el prioritario y cubrir todos los gastos de la familia. Como

1. Larrañaga, M. (2009). "Una oportunidad para impulsar cambios". Emakunde, 2009, ¿Cómo afecta la crisis económica a las mujeres? Monográfico de mujer y crisis editado por el Instituto Vasco de la Mujer, p. 11-13.

2. Castro, C. (2009). Economía sostenible: no sin igualdad de género. Emakunde, 2009, ¿Cómo afecta la crisis económica a las mujeres? Monográfico sobre mujer y crisis editado por el Instituto Vasco de la Mujer, p. 14-18. 
consecuencia de la depresión y posterior crisis de los 90 y, el aumento del desempleo masculino, fueron muchas las mujeres que se vieron empujadas a buscar trabajo. No obstante, la finalidad última de esta incorporación laboral, estaba lejos de ser la emancipación, más bien se consideraba como una situación forzosa ${ }^{3}$.

Esta percepción sobre el trabajo femenino como complementaria al del hombre desemboca en una desvalorización de los puestos de trabajo mayoritariamente cubiertos por mujeres, con mayor precariedad y segregación laboral.

\section{Segregación laboral}

La segregación ocupacional por sexos da lugar a la separación de los mercados de trabajo masculino y femenino, de modo que los hombres y mujeres se encuentran en distintas ramas de ocupación y/o distintas actividades. La problemática surge porque muchas de las veces las mujeres acaban situándose en los niveles más bajos del empleo en cuanto a cualificación y remuneración. Con ello, el concepto de segregación acaba implicando también discriminación. En principio el hecho de que los diferentes géneros se sitúen en lugares distintos de la escala ocupacional no resulta discriminatorio en sí mismo, pero lo es cuando las mujeres acaban por ocupar posiciones más desventajosas de un modo sistémico y, sin que ello, Ileve aparejado diferencias de cualificación por sexos o distintas preferencias a la hora de buscar trabajo ${ }^{4}$.

La segregación afecta negativamente tanto a los mercados de trabajo, debido a la rigidez que causa la movilidad entre ocupaciones masculinas y femeninas, como al colectivo femenino, al reducir sus oportunidades laborales generando diferencias de ingresos con respecto a los hombres ${ }^{5}$. Una teoría que permite explicar la segregación ocupacional entre hombre y mujeres es la de la segmentación de los mercados de trabajo. Uno de los modelos más conocidos se denomina "modelo de mercado de trabajo dual" propuesto por Doeringer y Piore en $1971^{6}$. Estos autores diferencian dos tipos de mercado de trabajo: uno primario y otro secundario. El primario responde a los empleos estables, con buena remuneración, con mejores condiciones de trabajo y con posibilidad de

3. Olalla, M., Morelló, A. y Álvarez, C. (2009). Efectos de la crisis actual en las mujeres trabajadoras de Cataluña y el estado español. Miembros pertenecientes a "Dones de Lluita Internacionalista". Jornadas feministas estatales. 30 años después: aquí y ahora. Granada, 5, 6, 7 de Diciembre.

4. Hidalgo, A., Pérez, S. y Calderón, M.J. (2007). La discriminación laboral de la mujer: una década a examen. Ministerio de Igualdad.

5. Maté, J. J., Nava, L. A. y Rodríguez Caballero, J.C. (2002). "La segregación ocupacional por razones de sexo en la economía española", Revista del Ministerio de Trabajo y Asuntos Sociales 36, pp. 79-94.

6. Doeringer, P.B. y Piore, M.J. (1971). Internal labor markets and manpower analysis. Lexington, Massachusetts: D.C Heath \& Company. Versión en castellano: Mercados internos de trabajo y análisis laboral. Ministerio de Trabajo y Asuntos Sociales. Madrid, 1985. 
promoción. Las empresas que ofrecen este tipo de empleo valoran mucho la experiencia dentro de la propia empresa y, por tanto, ofertan estos puestos a los varones que suelen tener menos interrupciones en su vida laboral. El mercado secundario está constituido por los empleos más inestables, peor pagados, con malas condiciones de trabajo y escasas posibilidad de promoción profesional. Las mujeres tenderán a ser excluidas del mercado primario para concentrarse en el secundario, creando la segregación ocupacional entre sexos y dividiendo al mercado en ocupaciones masculinas y femeninas.

Otra de las teorías económicas sugiere que la segregación ocupacional parte de factores de oferta y demanda. En los aspectos de demanda, se encuentra la discriminación de algunos empresarios o empleadores a la hora de contratar a una mujer, poniendo como escusa ejemplos como el de mayor absentismo laboral al recaer sobre ellas la mayor parte de las responsabilidades familiares, o al considerar que las mujeres están peor cualificadas, por lo que es habitual que las ocupaciones que requieren unos niveles de estudios elevados y en las que la experiencia en el puesto de trabajo cobra importancia, sean ofrecidas a hombres en mayor medida que a mujeres ${ }^{7}$. Tomando como punto de referencia la oferta existe una explicación basada en la teoría del capital humano que sugiere que la mujer se ve forzada a escoger trabajos compatibles con las tareas del hogar y/o las responsabilidades familiares (discriminación social), lo que hace que ocupe segmentos del mercado laboral con menor exigencia de cualificación, trabajos a tiempo parcial y de carácter temporal. Sea cual sea la perspectiva o teoría de análisis económica se llega a concluir que las mujeres presentan discriminación y mayor precariedad laboral.

\section{La precariedad laboral femenina se agrava en tiempos de crisis}

La precariedad laboral de la mujer se puede analizar a través de diferentes parámetros $^{8}$. A continuación se exponen alguno de ellos:

- En la situación de crisis actual el paro afecta tanto a mujeres como a hombres, aunque no en igual proporción debido a que los sectores de la construcción y la automoción, mayoritariamente con trabajadores varones, han sido las áreas más castigadas por la crisis. No obstante, conviene recordar que la situación de la mujer en el desempleo es peor que la de los hombres, están menos protegidas. Según el Ministerio de Trabajo, el número de parados

7. Dolado, J., Felgueroso, F. y Jimeno, J.F. (2003). "Where do woman work analysing patterns in occupational segregation by gender?". Annales Economie et de Statistique, pp. 71-84. Véase también Hidalgo, A., Pérez, S. y Calderón, M.J. (2007). La discriminación laboral de la mujer: una década a examen. Ministerio de Igualdad, p. 58.

8. Este tema puede ser consultado en: Larrañaga, M. (2009). "Una oportunidad para impulsar cambios", Emakunde; Olalla, M., Morelló, A. y Álvarez, C. (2009). Efectos de la crisis actual en las mujeres trabajadoras de Cataluña y el resto del estado; Hidalgo, A., Pérez, S. y Calderón, M.J. (2007). La discriminación laboral de la mujer: una década a examen. Ministerio de Igualdad. 
que percibe prestación supera al de paradas en mayor índice que la flecha de desempleo entre hombres y mujeres.

El incremento del paro ha hecho emerger una realidad en la que las mujeres ya no son mayoría en la composición del desempleo. El elemento más importante a destacar a partir del análisis de la composición y variabilidad del empleo y desempleo es el impacto de la formación en el mercado laboral, señalando una tendencia por la que los empleos más cualificados y las personas con mayores niveles de formación están protegiéndose con mayores garantías del paro. Ello significaría que en plena fase destructiva del empleo hay señales de evolución del tejido económico hacía un cambio del modelo productivo ${ }^{9}$.

Tabla 1: Distribución de la tasa de desempleo por sexo en el último trimestre del año 2010 y el último trimestre del 2004

\begin{tabular}{|c|c|c|c|c|}
\hline & $\begin{array}{c}\mathbf{4}^{\circ} \text { TRI. } \\
\text { AÑO 2010 }\end{array}$ & $\begin{array}{c}\text { VARI. PORC. } \\
\text { TRIMESTRE ANTE- } \\
\text { RIOR }\end{array}$ & $\begin{array}{c}\text { VARI. PORC. } \\
\text { IGUAL TRI. AÑO } \\
\text { ANTERIOR. }\end{array}$ & $\begin{array}{c}\mathbf{4}^{\mathbf{0}} \text { TRI. } \\
\text { ANNO 2004 }\end{array}$ \\
\hline TOTAL PARADOS & 4.645 .500 & $+0,71$ & $+12,28$ & $\mathbf{2 . 1 5 9 , 4}$ \\
\hline VARONES & 2.538 .000 & $-0,62$ & $+10,87$ & $\mathbf{9 3 1 , 5}$ \\
\hline MUJERES & $\mathbf{2 . 1 0 7 . 5 0 0}$ & $+\mathbf{2 , 3 6}$ & $+\mathbf{1 4 , 0 2}$ & $\mathbf{1 2 2 7 , 9}$ \\
\hline
\end{tabular}

FUENTE: A partir de los datos de INE-EPA ${ }^{10}$. Unidades: Miles de personas.

- Otro dato a tener en cuenta sobre la precariedad laboral es el empleo a tiempo parcial. Según datos del INE de los años 2008 y 2009 se aprecia como las jornadas de menos horas semanales corresponden a las mujeres ${ }^{11}$. La Comisión Europea recomendó a España aumentar los empleos a tiempo parcial con la finalidad de conciliar vida laboral y familiar. Esta medida, aparentemente, tuvo su éxito puesto que en el 2005 y tras años de estancamiento se produjo un incremento del $50 \%$ en la tasa de empleo de trabajos con este tipo de jornada. La ocupación de estos puestos de trabajo ha sido mayoritariamente femenina, puesto que las mujeres han Ilegado a cubrir el $80 \%$ de la oferta de este tipo ${ }^{12}$.

9. Homs, O. y Obeso, C. (2009)."Impacto de la crisis en las relaciones laborales en España". ICE: Revista de economía, 850, Primera crisis global: Procesos, consecuencias, medidas, pp. 89-108.

10. Datos obtenidos del Instituto Nacional de Estadística. Encuesta de población activa. Consultada el $4^{\text {a }}$ trimestre de 2010 en la sección de "parados por grupo de edad, sexo y sector económico".

11. Datos obtenidos del Instituto Nacional de Estadística. Encuesta de población activa. Consultada el $2^{\text {a }}$ trimestre de 2010 en la sección de "ocupados por tipo de contrato o relación laboral de los asalariados, sexo y sector económico".

12. De Lucas, F. y De la Cueva, M. (2009). "Crisis anunciada y sujeción de la mujer en el régimen familiarista". Portularia, IX (1), pp. 57-68. 
TABLA 2: Ocupados por número de horas efectivas trabajadas en el empleo principal por sexo

\begin{tabular}{|c|c|c|c|c|c|c|c|c|c|c|c|c|}
\hline \multirow[t]{2}{*}{ H: Horas } & \multicolumn{2}{|c|}{$1-9 \mathrm{H}$} & \multicolumn{2}{|c|}{$10-19 \mathrm{H}$} & \multicolumn{2}{|c|}{$20-29$ H } & \multicolumn{2}{|c|}{$39-39 \mathrm{H}$} & \multicolumn{2}{|c|}{$40-49 \mathrm{H}$} & \multicolumn{2}{|c|}{$500+$} \\
\hline & ஓे & 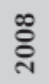 & ڤ્సે & 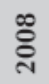 & $\stackrel{\text { }}{\stackrel{\text { Dे }}{ }}$ & $\stackrel{\text { ڤ̊ }}{\stackrel{2}{2}}$ & ஓ्̀े & 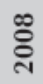 & ஓ्̀े & $\stackrel{\text { ڤ્ }}{\circ}$ & ஓ्̀े & $\stackrel{\infty}{\stackrel{\leftrightarrow}{\circ}}$ \\
\hline 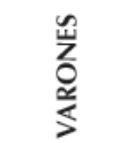 & รे & $\begin{array}{l}\circ \\
\text { ๙่ }\end{array}$ & $\begin{array}{l}+ \\
\stackrel{\circ}{-}\end{array}$ & $\begin{array}{l}\text { ñ } \\
\text { ڤ̊ }\end{array}$ & $\begin{array}{l}+ \\
\text { ப் } \\
\text { Lे }\end{array}$ & $\begin{array}{l}+ \\
\text { O' } \\
\text { Lे }\end{array}$ & 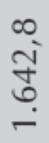 & శ & స̃ & חं & $\begin{array}{l}a \\
\frac{0}{6} \\
\text { !n }\end{array}$ & $\begin{array}{l}\infty \\
\text { 岌 } \\
\stackrel{1}{\Gamma} \\
\Gamma\end{array}$ \\
\hline 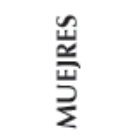 & $\begin{array}{l}\infty \\
\infty \\
\stackrel{\infty}{+\infty} \\
\text { స. }\end{array}$ & $\begin{array}{l}\stackrel{0}{J} \\
\stackrel{d}{J}\end{array}$ & $\stackrel{N}{N}$ & ๙ิ & 둘 & $\begin{array}{r}\text { g̊ } \\
\stackrel{+}{+} \\
-\end{array}$ & 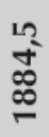 & $\begin{array}{l}7 \\
10 \\
10 \\
\infty\end{array}$ & $\begin{array}{l}0 \\
\infty \\
\infty \\
\infty \\
\text { ஸे }\end{array}$ & कृ & $\begin{array}{l}\circ \\
\text { ळे } \\
\dot{y}\end{array}$ & ָั \\
\hline
\end{tabular}

FUENTE: Datos obtenidos del INE-EPA. Unidades: Miles de personas.

No obstante, según información del CES del 2006, más de la mitad de las trabajadoras a tiempo parcial prefieren tener una jornada laboral completa. Sin embargo, únicamente el $8,8 \%$ de las mujeres que trabajan a tiempo completo buscan una jornada laboral a tiempo parcial ${ }^{13}$. Estos datos deben hacernos reflexionar sobre la situación de conciliación laboral y familiar de la mujer, tal como hoy está establecida. Deberíamos preguntarnos si es un rol "impuesto" socialmente y si en cierta medida las mujeres nos sentimos "forzadas" a desempeñarlo.

- La temporalidad laboral está emparejada con la precariedad del trabajo. En datos globales del 2009, obtenidos del INE, el 76,25 de los contratos indefinidos pertenecen a varones frente a un $72,7 \%$ de mujeres.

TABLA 3: Asalariados por sexo y tipo de contrato o relación laboral en valores absolutos ${ }^{14}$

\begin{tabular}{|l|c|c|c|c|}
\hline & \multicolumn{2}{|c|}{ VARONES } & \multicolumn{2}{c|}{ MUJERES } \\
\hline & 2009 & 2008 & 2009 & \multicolumn{1}{c|}{2008} \\
\hline CONTRATO INDEFINIDO & $8.493,7$ & $9.279,4$ & $7.187,0$ & $7.401,8$ \\
\hline CONTRATO TEMPORAL & $2.021,2$ & $2.559,4$ & $1.961,2$ & $2.321,1$ \\
\hline
\end{tabular}

13. Información del CES (Consejo Económico y Social de España). Consultado en la sección del comunicación, revistas y cauces en http://www.ces.es/index.jsp.

14. Datos obtenidos del Instituto Nacional de Estadística. Encuesta de población activa. Consultada el $2^{\mathrm{a}}$ trimestre de 2010 en la sección de "asalariados por sexo y tipo de contrato o relación laboral en valores absolutos". 
A las mujeres no solo les resulta más difícil obtener un contrato para salir del desempleo, sino que también tienen mayor dificultad para conseguirlo de modo indefinido, lo que produce mayor inestabilidad y precariedad a su inserción en el mercado de trabajo.

- La desigualdad retributiva es otro parámetro que forma parte de la discriminación y precariedad laboral femenina. Las mujeres cobran aproximadamente el $70 \%$ del salario de los hombres. Una discriminación salarial que en mayor o menor medida se encuentra en todos los grupos de edad, todos los niveles educativos, en todas los sectores de actividad, en las diferentes categorías profesionales y en todas las modalidades contractuales ${ }^{15}$. Una de las razones que explican estas diferencias salariales conecta directamente con el problema de la segregación ocupacional de la mujer, es decir, el salario recibido por un trabajador o trabajadora independientemente de su sexo, es menor en aquellas ocupaciones donde la presencia femenina es mayor ${ }^{16}$. El empleo de la mujer se localiza en porcentajes mayores al del hombre en las ramas de actividad y ocupaciones peor remuneradas.

Como anteriormente se ha apuntado, las mujeres tienen mayor presencia en las pequeñas y medianas empresas, ocupan de forma mayoritaria los empleos a tiempo parcial y los contratos temporales, donde reciben una remuneración más baja que los trabajadores de las grandes empresas, los que tienen contratos indefinidos y los que trabajan a tiempo completo. Inclusive y, agudizado por la crisis, en los puestos directivos la brecha salarial entre hombres y mujeres se sitúa en el 17\%, mientras que en las periodos pre-crisis lo hacía 5 puntos menos. Los mismo ocurre con la presencia femenina en este tipo de puestos que en los últimos años ha disminuido aproximadamente del 19 al $13 \%{ }^{17}$.

- Otro indicativo de precariedad laboral es el sub-empleo que, al igual que el paro, no ha dejado de crecer desde el inicio de la crisis. En España más de dos millones de personas se encuentran en esta situación. Este subempleo afecta de modo desigual a hombres y a mujeres, siendo estas últimas una vez más, el sexo desfavorecido. Según datos del $2^{\circ}$ trimestre de 2009 el 13\% de mujeres se declaran sub-ocupadas con respecto al $9 \%$ de hombres que afirman estar en esta situación ${ }^{18}$. Las épocas de bonanza económica agudizan la tendencia a la mercantilización de algunos trabajos domésticos y de cuidados, mientras que las épocas de crisis producen un giro y las mujeres desempleadas realizan este tipo de tareas en su propio hogar. De este modo, reducen gastos

15. Hidalgo, A., Pérez, S. y Calderón, M.J. (2007). La discriminación laboral de la mujer: una década a examen.

16. Salaberría, A. y Ullibarri, M. (1997). ¿Trabajar en ocupaciones femeninas reduce los salarios? Documento de trabajo de la Universidad Pública de Navarra DT 9706. Mayo 1997.

17. Datos obtenidos en http://www.losrecursoshumanos.com en el artículo La crisis refuerza la brecha salarial entre hombres y mujeres en los puestos directivo. Agencia Europa Press. Consultado [08/10/2010].

18. Larrañaga, M. (2009). Una oportunidad para impulsar cambios. 
familiares realizando labores que en épocas anteriores estaban remunerados, pero a costa de un aumento de trabajo no cotizado que continúa recayendo de forma mayoritaria en el género femenino.

\section{Medidas gubernamentales ante la crisis}

El efecto de la crisis económica sobre el mercado de trabajo ha provocado por parte de los Gobiernos la aprobación de planes de gasto público dirigidos, en gran medida, a fortalecer el modo familiarista vigente, en el que el hombre es el agente proveedor económico y la mujer la responsable de los cuidados. En esta línea y con el propósito de reactivar la economía, los gobiernos han priorizado como medidas anti-crisis, además de polémicos recortes salariales y propuestas en el aumento de la edad de jubilación, impulsar el gasto público en la recuperación del empleo masculino destruido, especialmente en los sectores de la construcción y del automóvil, así como asegurar y ampliar las prestaciones por desempleo a las personas afectadas por los expedientes de regulación ${ }^{19}$. Mientras que estas medidas anti-crisis afectan mayoritariamente a hombres en el intento de reincorporarles en el mercado de trabajo, los Gobiernos han tomado otra serie de medidas que están afectado desfavorablemente y de forma mayoritaria a mujeres, dificultando su inserción en el empleo de calidad y sus independencia económica, como son: el fomento de la reducción de la jornada y la contratación a tiempo parcial.

Si es cierto que el propósito de reactivar la economía mediante el gasto público en infraestructuras físicas puede ser beneficioso, no lo es menos que impulsar empleos en servicios sociales, atención a la dependencia o educación, además de incidir de un modo directo en el bienestar social, ampliaría el mercado laboral español "históricamente femenino".

Está claro que el panorama de mercado laboral de la época pre-crisis va a diferenciarse sustancialmente con el de años posteriores, pero la pregunta que nos debemos hacer es si los Gobiernos y Autoridades Competentes van a aprovechar estos momentos de reestructuración obligada para crear un mercado de trabajo menos desigual en términos de segregación, de salarios, de precariedad, que ayude a atenuar los roles de género.

\section{Crítica feminista de las medidas anti-crisis}

Como anteriormente se ha apuntado, el paro afecta en mayor medida a los hombres que a las mujeres lo que aumenta el número de familias que dependen económicamente en exclusiva del salario femenino. Esta nueva situación

19. Sobre el tema, véase Plataforma feminista contra la crisis. (2009), Salir del túnel con perspectiva feminista, Emakunde, Monográfico dedicado a mujer y crisis, ¿Cómo afecta la crisis económica a las mujeres?, pp. 18-21. 
deteriora la economía familiar que pasa de depender de dos sueldos a uno solo y mayoritariamente el menor de ellos. Ante este aumento de desempleo masculino cabe analizar si esto conlleva cambios en los roles familiares y de género, como por ejemplo, una participación más activa de los hombres en los trabajos no remunerados. Hasta ahora, los datos señalan que los varones que han pasado de una situación de ocupación a una de desempleo aumentan mínimamente la dedicación a trabajos no mercantiles, mientras que las mujeres que se ven en la misma situación prácticamente duplican la dedicación a las ocupaciones del hogar y cuidados ${ }^{20}$. Es obvio que, mientras que no exista un reparto de las labores no remuneradas entre hombre y mujeres, es difícil que las desigualdades laborales desaparezcan.

El Gobierno de España, a través de la Ley de Igualdad ha promovido medidas con el fin de que la mujer tenga las mismas condiciones que el hombre en el ámbito laboral. Sin embargo, en tiempo de crisis, los planes que las empresas tienen que diseñar para atenuar las desigualdades laborales en cuestión de género se han quedado únicamente en el papel, sin llegar a ejecutarse ${ }^{21}$. Esta Ley no es más que el punto de partida que debe ser completado con un cambio de paradigma. Llegar a la conclusión de que la mujer ni puede, ni debe, ni quiere, responsabilizarse sola de la familia y asumir dos roles a la vez para poder tener igualdad de oportunidades. Las tareas no remuneradas, especialmente las tareas del hogar y cuidados, deben ser compartidas.

Las autoridades nacionales e internacionales coinciden en valorar la igualdad de género como una estrategia rentable y económicamente eficaz. Sin embargo, en la práctica las respuestas político-económicas anti-crisis se han olvidado de ello. Está demostrado que la igualdad de género es clave para el desarrollo sostenible y, que cualquier otro intento que obvie esta premisa significaría seguir manteniéndonos dentro del mismo sistema que nos ha abocado a esta situación. En este sentido ante la crisis el feminismo tiene mucho que decir, porque representa los intereses, por lo menos, del cincuenta por ciento de la humanidad ${ }^{22}$.

Como ya se ha comentado, el Gobierno Español ha reaccionado desde la inercia de "salvar el sistema actual". En este sentido, el fondo de Inversión Local de 2009 se caracterizó por la ausencia total de inversiones en infraestructuras sociales, a pesar de que éstas hacen falta para atender las necesidades de cuidados de infancia y personas dependientes. En 2010 se aprobó un nuevo Fondo de Inversión Local con objeto de financiar proyectos municipales

20. Larrañaga, M. (2009). Una oportunidad para impulsar cambios.

21. Opinión obtenida de Sánchez-Silva, en el artículo publicado en http://www.elpais. com el [08/03/2009] y titulado La crisis amenaza la revolución de la mujer. [Consultado el 10/10/2010].

22. Falcó, L. (2010). "Feminismo en tiempos de crisis". En Clavo, M. J. y Goicoechea, M. A. (coord.). Miradas multidisciplinares para un mundo en igualdad: Ponencias de la I Reunión Científica sobre lgualdad y Género. 
de desarrollo sostenible relacionados con el medio ambiente, la innovación económica y las iniciativas sociales. Sin embargo, esta medida carece de un informe de impacto de género, por lo que no se aprecia un cambio sustancial con la medida del anterior $\mathrm{año}^{23}$.

Esta crisis podría haber representado una oportunidad para impulsar cambios al dejar patente que el sistema económico basado en un modelo de crecimiento indefinido en un mundo de recursos limitados ha fracasado. En este sentido, las Instituciones Competentes no deberían haber intentado reactivar el sistema que produjo la actual crisis a toda costa, sino analizar la situación como una oportunidad para forjar un futuro más igualitario y sostenible, considerando qué es lo que se contabiliza dentro del PIB y cómo otras producciones que quedan fuera de este cómputo deberían ser fomentadas, como son el bienestar social y el ámbito familiar ${ }^{24}$.

En resumen, la inversión pública masiva, necesaria para crear empleo y reactivar la economía, debería instrumentalizarse como una oportunidad única para eliminar la división sexual del trabajo, universalizando los servicios públicos de educación infantil y atención a la dependencia, mejorando el sistema de salud, de educación, de Servicios Sociales, promoviendo la igualdad en el deporte y la cultura ${ }^{25}$.

\section{Mujeres educadoras, crisis y cambios de roles}

En la división tradicional del trabajo y de los roles sociales, los hombres históricamente, tenían mayor presencia en la esfera pública que, por otro lado, está socialmente mejor valorada que la privada. En cambio, las mujeres quedaron relegadas al mundo de lo personal y, al mantenimiento y desarrollo de unas determinadas cualidades consideradas especialmente femeninas. De este modo, se invisibiliza la contribución femenina el desarrollo social ${ }^{26}$.

Actualmente y en cierta medida, debido al cambio en el mercado laboral provocado por la crisis, la mujer aumenta su presencia en las tasas de ocupación con respecto a los hombres mientras éstos la reducen por el incremento

23. Castro, C. Economía sostenible: no sin igualdad de género. Larrañaga, M. (2009). Una oportunidad para impulsar cambios.

24. Larrañaga, M. (2009). Una oportunidad para impulsar cambios.

25. Plataforma feminista contra la crisis. (2009). Salir del túnel con perspectiva feminista., p. 15.

26. Blanco, P. (2004). "La perspectiva de género, una necesidad en la construcción de la ciudadanía: Algunas actividades en la formación del profesorado", en M. I. Vera (2004). Formación de la ciudadanía: las TICs y los nuevos problemas, pp. 1-12. Sobre este tema véase a Subirats, M. (1994). "Conquistar la igualdad: la coeducación hoy", Revista Iberoamericana de Educación 6, pp. 49-78. También de esta autora el libro Balones fuera: reconstruir los espacios desde la coeducación. 
del desempleo masculino. Este cambio "forzoso" de roles debería ser el inicio de la equidad tanto en los trabajos remunerados como los propios del hogar y los cuidados. Las instituciones educativas deben aprovechar el momento para que las mujeres y hombres del mañana vivan esta situación como normalizada y no como fruto de un contexto de inestabilidad económica.

Los avances conseguidos por las mujeres en su lucha en pro de la igualdad han sido notables. En este camino la educación es y debe ser una pieza fundamental. No obstante, las instituciones educativas siguen actuando, aunque no siempre de forma intencionada, como un lugar de reproducción de los sistemas de género ${ }^{27}$. Teniendo en cuenta que la función docente está de forma masiva en manos de mujeres, esto supone la paradoja de que somos nosotras mismas las que reproducimos y transmitimos los patrones culturalmente heredados, que en muchos casos deseamos combatir.

La educación debe posicionarse en una postura que corrija las desigualdades y, esto debe hacerse a lo largo de todas las etapas educativas. No podemos cambiar esta situación confiando exclusivamente en la asignatura de Educación para la Ciudadanía, donde "supuestamente" se tratan cuestiones de género. La educación para la igualdad dentro del Sistema Educativo tiene que tener una visión amplia e integral. La prácticas escolares deben utilizar fórmulas como: la revisión de los contenidos sexistas del currículo, la utilización de un lenguaje menos discriminatorio, la eliminación de roles y estereotipos, la visibilización de la contribución histórica de la mujer, ser conscientes de la existencia de un currículo oculto en intento de combatirlo. La educación para la igualdad debe cubrir el itinerario escolar completo y debe implicar, no sólo los aspectos relativos a la docencia, sino el propio funcionamiento de los centros y la participación de toda la Comunidad Educativa. En definitiva, se necesita una modificación del Sistema para que los estereotipos y roles sexuales que se transmiten a las nuevas generaciones sean modificados respecto a los actuales, por tanto, contribuyan a crear una sociedad más igualitaria. Dentro de esta modificación no se debe olvidar la formación del profesorado como pieza fundamental para introducir la coeducación en el currículo. Para ello, se debe sensibilizar al profesorado sobre su papel activo en la Educación de Igualdad de oportunidades entre sexos, formándoles sobre los efectos de la socialización de género, favoreciendo la reflexión y la crítica de las actuaciones docentes que refuerzan y afianzan los estereotipos y roles de género en la escuela e, introducir experiencias y materiales didácticos que les posibilite educar activamente en la igualdad a las alumnas y alumnos ${ }^{28}$.

Si pretendemos que el alumnado que en este momento forma parte del Sistema Educativo aprenda a construir sus relaciones tanto personales como

27. Acker, S. (1997). Género y educación: Reflexiones sociológicas sobre mujeres, enseñanza y feminismo. Madrid: Narcea. Véase también Bonal, X. (1997). Las actitudes del profesorado ante la coeducación: propuestas de intervención. Barcelona: Grao.

28. Blanco, P. (2004). La perspectiva de género, una necesidad. 
sociales desde el respeto y sean capaces de diseñar un futuro sin asimetrías discriminatorias para que los hombres y mujeres del futuro ocupen por igual los espacios públicos y privados, se les debe dar criterios y fundamentos. La educación debe aprovechar el momento en el que los roles sociales están cambiando "forzosamente" para instrumentalizar esta situación trasladándola a las aulas. Debe mostrar esta realidad como algo a potenciar y no como la consecuencia de la crisis, de modo que, una vez superada ésta, no se restablezcan los roles tradicionales. 